\title{
Fiber Bragg Grating (FBG) sensors as flatness and mechanical stretching sensors
}

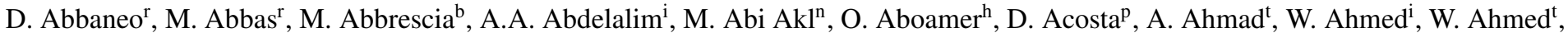

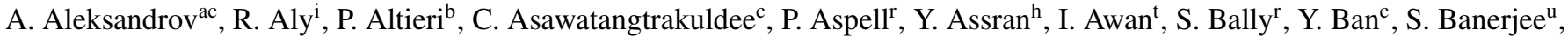

V. Barashko ${ }^{\mathrm{p}}$, P. Barria ${ }^{\mathrm{e}}$, G. Bencze ${ }^{\mathrm{g}}$, N. Beni ${ }^{\mathrm{k}}$, L. Benussi ${ }^{\mathrm{o}}$, V. Bhopatkar ${ }^{\mathrm{x}}, \mathrm{S}$. Bianco ${ }^{\mathrm{o}}$, J. Bos ${ }^{\mathrm{r}}$, O. Bouhali ${ }^{\mathrm{n}}$, A. Braghieriaa ${ }^{\mathrm{a}}$, S. Braibant ${ }^{\mathrm{d}}$,

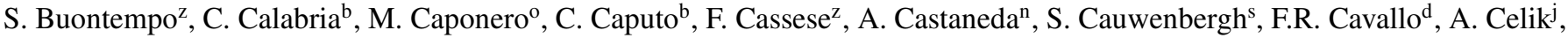
M. Choi ${ }^{\mathrm{ag}}$, S. Choi ${ }^{\mathrm{ae}}$, J. Christiansen ${ }^{\mathrm{r}}$, A. Cimmino ${ }^{\mathrm{s}}$, S. Colafranceschi ${ }^{\mathrm{r}}$, A. Colaleo ${ }^{\mathrm{b}}$, A. Conde Garcia ${ }^{\mathrm{r}}$, S. Czellar ${ }^{\mathrm{k}}$, M.M. Dabrowski ${ }^{\mathrm{r}}$, G. De Lentdecker ${ }^{\mathrm{e}}$, R. De Oliveira ${ }^{\mathrm{r}}$, G. de Robertis ${ }^{\mathrm{b}}$, S. Dildick $\mathrm{j}^{\mathrm{j}, \mathrm{s}}$, B. Dorney ${ }^{\mathrm{r}}$, W. Elmetenawee ${ }^{\mathrm{i}}$, G. Endroczi ${ }^{\mathrm{g}}$, F. Errico ${ }^{\mathrm{b}}$, A. Fenyvesi ${ }^{\mathrm{k}}$, S. Ferry ${ }^{\mathrm{r}}$, I. Furic ${ }^{\mathrm{p}}$, P. Giacomelli ${ }^{\mathrm{d}}$, J. Gilmore ${ }^{\mathrm{j}}$, V. Golovtsov ${ }^{\mathrm{q}}$, L. Guiducci ${ }^{\mathrm{d}}$, F. Guilloux ${ }^{\text {ab }}$, A. Gutierrez ${ }^{\mathrm{m}}$, R.M. Hadjiiska ${ }^{\text {ac }}$, A. Hassan ${ }^{\mathrm{i}}$,

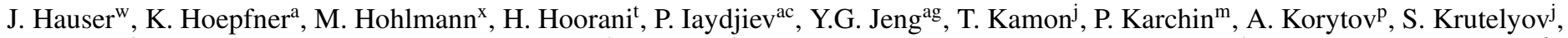

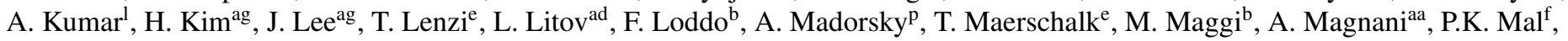
K. Mandal ${ }^{\mathrm{f}}$, A. Marchioror ${ }^{\mathrm{r}}$, A. Marinov ${ }^{\mathrm{r}}$, R. Masod ${ }^{\mathrm{h}}$, N. Majumdar ${ }^{\mathrm{u}}$, J.A. Merlin, ${ }^{\mathrm{r}, a h}$, G. Mitselmakher ${ }^{\mathrm{p}}$, A.K. Mohanty ${ }^{\mathrm{y}}$, S. Mohamed ${ }^{\mathrm{h}}$, A. Mohapatra ${ }^{\mathrm{x}}$, J. Molnar ${ }^{\mathrm{k}}$, S. Muhammad ${ }^{\mathrm{t}, \mathrm{o}}$, S. Mukhopadhyay ${ }^{\mathrm{u}}$, M. Naimuddin' ${ }^{1}$, S. Nuzzo ${ }^{\mathrm{b}}$, E. Oliveri ${ }^{\mathrm{r}}$, L.M. Pant ${ }^{\mathrm{y}}$, P. Paolucci ${ }^{\mathrm{z}}$, I. Park ${ }^{\mathrm{ag}}$, G. Passeggio ${ }^{\mathrm{z}}$, L. Passamonti ${ }^{\mathrm{O}}$, B. Pavlov ${ }^{\mathrm{ad}}$, B. Philipps ${ }^{\mathrm{a}}$, D. Piccolo ${ }^{\mathrm{o}}$, D. Pierluigi ${ }^{\mathrm{o}}$, H. Postema ${ }^{\mathrm{r}}$, A. Puig Baranac ${ }^{\mathrm{r}}$, A. Radi ${ }^{\mathrm{h}}$, R. Radogna ${ }^{\mathrm{b}}$, G. Raffone ${ }^{\mathrm{o}}$, A. Ranieri ${ }^{\mathrm{b}}$, G. Rashevski ${ }^{\mathrm{ac}}$, C. Riccardi $^{\mathrm{aa}}$, M. Rodozov ${ }^{\mathrm{ac}}$, A. Rodrigues ${ }^{\mathrm{r}}$, L. Ropelewski $^{\mathrm{r}}$, S. RoyChowdhury ${ }^{\mathrm{u}}$, A. Russo ${ }^{\circ}$, G. Ryu ${ }^{\text {ag }}$, M.S. Ryu ${ }^{\text {ag }}$, A. Safonov ${ }^{j}$, S. Salva ${ }^{\mathrm{s}}$, G. Saviano ${ }^{\circ}$, A. Sharma ${ }^{\text {b }}$, A. Sharma ${ }^{\mathrm{r}}$, R. Sharma ${ }^{1}$, A.H. Shah ${ }^{\mathrm{l}}$, M. Shopovac, J. Sturdy ${ }^{\mathrm{m}}$, G. Sultanov ${ }^{\mathrm{ac}}$, S.K. Swain ${ }^{\mathrm{f}}$, Z. Szillasi ${ }^{\mathrm{k}}$, J. Talvitie ${ }^{\mathrm{v}}$, A. Tatarinov ${ }^{\mathrm{j}}$, T. Tuuva ${ }^{\mathrm{v}}$, M. Tytgat ${ }^{\mathrm{s}}$, I. Vai ${ }^{\text {aa }}$, M. Van Stenis ${ }^{\mathrm{r}}$, R. Venditti ${ }^{\mathrm{b}}$,

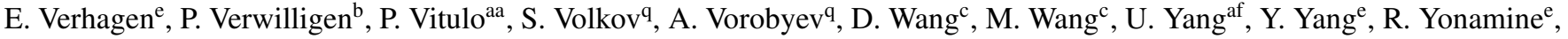
N. Zaganidis', F. Zenoni ${ }^{\mathrm{e}}$, A. Zhang ${ }^{\mathrm{x}}$

${ }^{a}$ RWTH Aachen University, III Physikalisches Institut A, Aachen, Germany

${ }^{b}$ INFN Bari and University of Bari, Bari, Italy ${ }^{c}$ Peking University, Beijing, China

${ }^{d}$ INFN Bologna and University of Bologna, Bologna, Italy

${ }^{e}$ Universite Libre de Bruxelles, Brussels, Belgium

${ }^{f}$ National Institute of Science Education and Research, Bhubaneswar

${ }^{g}$ Institute for Particle and Nuclear Physics, Wigner Research Centre for Physics, Hungarian Academy of Sciences, Budapest, Hungary

${ }^{h}$ Academy of Scientific Research and Technology - Egyptian Network of High Energy Physics, ASRT-ENHEP, Cairo, Egypt ${ }^{i}$ Helwan University \& CTP, Cairo, Egypt

${ }^{j}$ Texas AEM University, College Station, U.S.A.

${ }^{k}$ Institute for Nuclear Research of the Hungarian Academy of Sciences (ATOMKI), Debrecen, Hungary ${ }^{l}$ University of Delhi, Delhi, India

${ }^{m}$ Wayne State University, Detroit, U.S.A

${ }^{n}$ Texas AE M University at Qatar, Doha, Qatar

${ }^{o}$ Laboratori Nazionali di Frascati - INFN, Frascati, Italy

${ }^{p}$ University of Florida, Gainesville, U.S.A.

${ }^{q}$ Petersburg Nuclear Physics Institute, Gatchina, Russia ${ }^{r}$ CERN, Geneva, Switzerland

${ }^{s}$ Ghent University, Dept. of Physics and Astronomy, Ghent, Belgium

${ }^{t}$ National Center for Physics, Quaid-i-Azam University Campus, Islamabad, Pakistan

${ }^{u}$ Saha Institute of Nuclear Physics, Kolkata, India

${ }^{v}$ Lappeenranta University of Technology, Lappeenranta, Finland

${ }^{w}$ University of California, Los Angeles, U.S.A.

${ }^{x}$ Florida Institute of Technology, Melbourne, U.S.A.

${ }^{y}$ Bhabha Atomic Research Centre, Mumbai, India ${ }^{z}$ INFN Napoli, Napoli, Italy

${ }^{a}$ INFN Pavia and University of Pavia, Pavia, Italy

${ }^{a b}$ IRFU CEA-Saclay, Saclay, France

${ }^{a c}$ Institute for Nuclear Research and Nuclear Energy, Sofia, Bulgaria

${ }^{a d}$ Sofia University, Sofia, Bulgaria

${ }^{a e}$ Korea University, Seoul, Korea

${ }^{a f}$ Seoul National University, Seoul, Korea

${ }^{a g}$ University of Seoul, Seoul, Korea

${ }^{a h}$ Institut Pluridisciplinaire - Hubert Curien (IPHC), Strasbourg, France

\begin{abstract}
A novel approach which uses Fibre Bragg Grating (FBG) sensors has been utilised to assess and monitor the flatness of Gaseous Electron Multipliers (GEM) foils. The setup layout and preliminary results are presented.
\end{abstract}

Keywords: FBG sensors, Triple-GEM detector, Mechanical stretching, foils planarity, gas detectors PACS: 29.40.Cs, 29.40.Gx 


\section{FBG sensors as a strain measurement}

To upgrade the Compact Muon Solenoid (CMS) muon system ${ }^{58}$ 144 GEM chambers will be installed in the high pseudorapidity re- 59 gion of CMS during Long Shutdown 2 (LS2) of the Large Hadron Collider [1]. The GEMs can provide extra leverage on precision studies of standard model physics, as well as open up a window to explore exotic signatures with muons in the high eta region [2]. The GEM chambers will be located close to the beam pipe where a high flux of low Pt muons is expected. The GEM chambers can easily handle this rate due to their high rate capability of $100 \mathrm{MHz} / \mathrm{cm}^{2}$. The large active area of each GE1/1 (GEM Endcap) chamber, approximately $0.4 \mathrm{~m}^{2}$ [3], consists of a triple-GEM foil stack. These foils need to be stretched simultaneously in order to secure the planarity and consequent uniform performance of the GE1/1 chamber [4]. The GE1/1 detector technology used for CMS is described in detail in these same conference proceedings (Elba 2015) by Gilles De Lentdecker with title "Status Report of the Upgrade of the CMS 60 muon system with triple-GEM detectors". The FBG sensors act 61 as low cost precision spatial and temperature sensing tools and they 62 are commonly used for strain measurements [5] [6] [7]. In this work 63 FBG sensors are used to measure the planarity and mechanical ten- 64 sion of the GEM foils in the GE1/1 chambers. A FBG is a type of 65 distributed Bragg reflector, constructed in a short segment of optical fiber that reflects particular wavelengths of light and transmits all others. The sensitivity of FBG in terms of strain, defined as relative elongation w.r.t. the initial position is of the order of 0.1 micron. ${ }_{67}$ This is achieved by creating a periodic variation in the refractive in- ${ }_{68}$ dex of the fiber core, which generates a wavelength-specific dielec- ${ }_{69}$ tric mirror. Therefore it can be used as a strain measurement tool ${ }_{70}$ since variation of the FBG translates into different light frequency ${ }_{71}$ response. In order to validate the mechanical stretching technique 72 a network of FBG sensors is affixed on the triple-GEM stack. Each sensor is glued on the GEM foil using a very thin layer of epoxy ${ }_{74}$ glue. The test is performed by modifying the stretching conditions of the GEM foils stack with real time monitoring and recording of the FBG sensors data. The test starts with the chamber normally as- ${ }^{75}$ sembled with the GEM stack mechanically stretched to the nominal tensile load. After some time, when the initial conditions are stabi- ${ }^{76}$ lized, the mechanical stretching of the GEMs is released and kept ${ }^{77}$ in such condition for several hours. Finally the GEMs are stretched again up to the nominal tensile load. The trends of the FBG sensors are shown in figure 1(Left). The steep variations of the strain evident in figure 1(Left) correspond to the actions of un-screwing 80 and screwing the mechanical stretchers during the test. The initial stretch value is assumed as reference condition with strain $=0{ }_{82}^{81}$ When stretchers are un-screwed the strain goes to the lower value, ${ }_{83}$ different strain values apply to different foils as they fold quasi-free 84 and assume unequal conditions. After the stretchers are screwed ${ }^{85}$ back, the strain value is similar for all foils, showing that they all ${ }_{87}^{86}$ experience similar stretching, about the original value of the refer- 88 ence condition. Thus it can be inferred that at the predetermined ${ }^{89}$ tensile load all foils reach a similar stretched level although they ${ }_{91}^{90}$ started from different values. From the plot it can be seen that all ${ }_{92}^{91}$ the sensors of the network react at the same moment. These results 93 allow us to validate the mechanical stretching assembly technique ${ }^{94}$

\footnotetext{
${ }^{*}$ Corresponding author

Email addresses: luigi.benussi@lnf .infn.it (L. Benussi), saleh.muhammad@cern.ch (S. Muhammad)
}

for GE1/1 chambers. Further tests are ongoing to confirm other important parameters such as the optimal tensile load to be applied to the GEMs and the maximum planarity obtainable for the GEMs without applying a load beyond the Young's region for GEM foils.
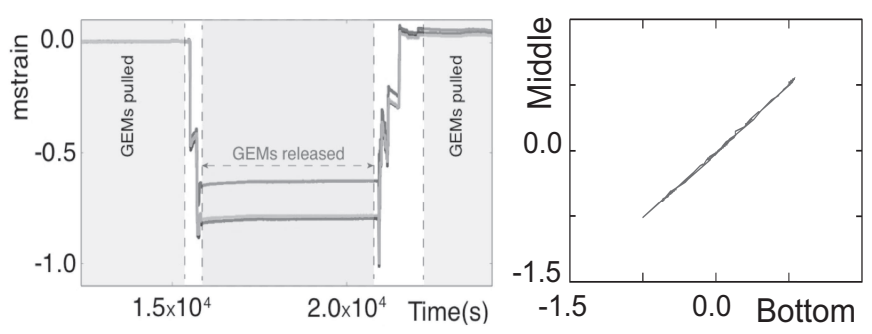

Figure 1: (Left)Three regions corresponding to the mechanical stretched, loose and again stretched triple GEM foils stack respectively. (Right) The correlation of the strains measured in two different foils of the stack

In figure 1(Right), the mutual comparison of two GEM foils (the bottom and the middle ones) shows the almost perfect correlation between the two strian measured demonstrating that all the foils realize almost the same strain during the assembly. This shows that the adopted stretching technique is validated at nominal tensile stress.

\section{Conclusion}

By using the FBG sensors we successfully demonstrated that the mechanical stretching technique adopted to assemble the GE1/1 chambers is reliable and secures the correct tensioning of the three foils. By applying the correct tension across the GEM stack a uniform gap spacing can be obtained, which is extremely important to get the required performance of the detector. Several tests are ongoing by using the same FBG sensors to optimize the tensile load in order to avoid damage and guarantee planarity of the GEM foils.

\section{Acknowledgments}

We gratefully acknowledge the support of FRS-FNRS (Belgium), FWO-Flanders (Belgium), BSF-MES (Bulgaria), BMBF (Germany), DAE (India), DST (India), INFN (Italy), NRF (Korea), QNRF (Qatar), and DOE (USA).

\section{References}

[1] D. Abbaneo et al., Performance of a Large-Area GEM Detector Prototype for the Upgrade of the CMS Muon Endcap System, arXiv:1412.0228v2, 8 Dec 2014.

[2] D. Abbaneo et al., Upgrade of the CMS muon system with tripleGEM detectors, JINST 9 (2014) C10036, http://iopscience.iop.org/17480221/9/10/C10036/.

[3] A. Colaleo et al., CMS TECHNICAL DESIGN REPORT FOR THE MUON ENDCAP GEM UPGRADE, CERN-LHCC-2015-012. CMS-TDR013, https://cds.cern.ch/record/2021453.

[4] D. Abbaneo et al.,Status of the Triple-GEM project for the upgrade of the CMS Muon System, DOI: 10.1088/1748-0221/8/12/C12031.

[5] L. Benussi et al.,The Omega-like: A novel device using FBG sensors to position vertex detectors with micrometric precision, Nucl.Phys.Proc.Suppl. 172.

[6] M. Caponero et al., Use of fiber optic technology for relative humidity monitoring in RPC detectors, Published in PoS RPC2012 (2012) 073.

[7] L. Benussi et al., A Novel Temperature Monitoring Sensor for Gas-Based Detectors in Large HEP Experiments, doi "http://dx.doi.org/10.1016/j.phpro.2012.02.400" 